\title{
Remarks on inequalities for the Casorati curvatures of slant submanifolds in quaternionic space forms
}

Pan Zhang and Liang Zhang*

\section{*Correspondence:} zhliang43@163.com School of Mathematics and Computer Science, Anhui Normal University, Huajing South Road, Yijiang District, Wuhu, Anhui 241000, P.R. China

\begin{abstract}
In this paper, we obtain an inequality for the normalized Casorati curvature of slant submanifolds in quaternionic space forms by using T Oprea's optimization method. MSC: 53C40; 53D12
\end{abstract}

Keywords: inequalities; Casorati curvatures; quaternionic space forms

\section{Introduction}

The Casorati curvature of an $n$-dimensional submanifold $M$ of a Riemannian manifold, usually denoted by $\mathcal{C}$, is an extrinsic invariant defined as the normalized square of the length of the second fundamental form of the submanifold. In [1], Decu et al. introduced the normalized $\delta$-Casorati curvatures $\delta_{c}(n-1)$ and $\hat{\delta}_{c}(n-1)$ by

$$
\left[\delta_{c}(n-1)\right]_{x}=\frac{1}{2} \mathcal{C}_{x}+\frac{n+1}{2 n(n-1)} \inf \left\{\mathcal{C}(L) \mid L \text { a hyperplane of } T_{x} M\right\}
$$

and

$$
\left[\hat{\delta}_{c}(n-1)\right]_{x}=2 \mathcal{C}_{x}-\frac{2 n-1}{2 n} \sup \left\{\mathcal{C}(L) \mid L \text { a hyperplane of } T_{x} M\right\}
$$

where $x \in M$, and established some inequalities involving these invariants for submanifolds in real space forms. Later, Slesar et al. proved two inequalities relating the above normalized Casorati curvatures for a slant submanifolds in a quaternionic space form in [2]. However, it was pointed out that the coefficient $\frac{n+1}{2 n(n-1)}$ in (1) is inappropriate and must be replaced by $\frac{n+1}{2 n}[3,4]$. Following [3, 4], we define the normalized $\delta$-Casorati curvature $\delta_{C}(n-1)$ by

$$
\left[\delta_{C}(n-1)\right]_{x}=\frac{1}{2} \mathcal{C}_{x}+\frac{n+1}{2 n} \inf \left\{\mathcal{C}(L) \mid L \text { a hyperplane of } T_{x} M\right\} .
$$

By using T Oprea's optimization method on Riemannian submanifolds, we establish the following inequalities in terms of $\delta_{C}(n-1)$ for $\theta$-slant proper submanifolds of a quaternionic space form.

O2014 Zhang and Zhang; licensee Springer. This is an Open Access article distributed under the terms of the Creative Commons Attribution License (http://creativecommons.org/licenses/by/2.0), which permits unrestricted use, distribution, and reproduction in any medium, provided the original work is properly cited. 
Theorem 1 Let $M^{n}, n \geq 3$, be $\theta$-slant proper submanifold of a quaternionic space form $\bar{M}^{4 m}(c)$. Then the normalized $\delta$-Casorati curvature $\delta_{C}(n-1)$ satisfies

$$
\rho \leq \delta_{C}(n-1)+\frac{c}{4}\left(1+\frac{9}{n-1} \cos ^{2} \theta\right)
$$

where $\rho$ is the normalized scalar curvature of $M^{n}$. Moreover, the equality case holds if and only if $M^{n}$ is an invariantly quasi-umbilical submanifold with trivial normal connection in $\bar{M}^{4 m}(c)$, such that with respect to suitable orthonormal tangent frame $\left\{\xi_{1}, \ldots, \xi_{n}\right\}$ and normal orthonormal frame $\left\{\xi_{n+1}, \ldots, \xi_{4 m}\right\}$, the shape operators $A_{r}=A_{e_{r}}, r \in\{n+1, \ldots, 4 m\}$, take the following forms:

$$
A_{n+1}=\left(\begin{array}{cccccc}
a & 0 & 0 & \cdots & 0 & 0 \\
0 & a & 0 & \cdots & 0 & 0 \\
0 & 0 & a & \cdots & 0 & 0 \\
\vdots & \vdots & \vdots & \ddots & \vdots & \vdots \\
0 & 0 & 0 & \cdots & a & 0 \\
0 & 0 & 0 & \cdots & 0 & 2 a
\end{array}\right), \quad A_{n+2}=\cdots=A_{4 m}=0
$$

\section{Preliminaries}

Let $\left(M^{n}, g\right)$ be an $n$-dimensional submanifold in an $(n+p)$-dimensional Riemannian manifold $\left(\bar{M}^{n+p}, \bar{g}\right)$. The Levi-Civita connections on $\bar{M}^{n+p}$ and $M^{n}$ will be denoted by $\bar{\nabla}$ and $\nabla$, respectively. For all $X, Y \in C^{\infty}(T M), N \in C^{\infty}\left(T M^{\perp}\right)$, the Gauss and Weingarten formulas can be expressed by

$$
\bar{\nabla}_{X} Y=\nabla_{X} Y+h(X, Y), \quad \bar{\nabla}_{X} N=-A_{N} X+\nabla_{X}^{\perp} N
$$

where $h$ is the second fundamental form of $M, \bar{\nabla}$ is the normal connection and the shape operator $A_{N}$ of $M$ is given by

$$
g\left(A_{N} X, Y\right)=\bar{g}(h(X, Y), N) .
$$

The submanifold $M$ is said to be totally geodesic if $h=0$. Besides, $M$ is called invariantly quasi-umbilical if there exist $p$ mutually orthogonal unit normal vectors $\xi_{n+1}, \ldots, \xi_{n+p}$ such that the shape operators with respect to all directions $\xi_{r}$ have an eigenvalue of multiplicity $n-1$ and that for each $\xi_{r}$ the distinguished eigendirection is the same [1-4].

In $\bar{M}^{n+p}$ we choose a local orthonormal frame $e_{1}, \ldots, e_{n}, e_{n+1}, \ldots, e_{n+p}$, such that, restricting ourselves to $M^{n}, e_{1}, \ldots, e_{n}$ are tangent to $M^{n}$. We write $h_{i j}^{r}=g\left(h\left(e_{i}, e_{j}\right), e_{r}\right)$. Then the mean curvature vector $H$ is given by

$$
H=\sum_{r=n+1}^{n+p}\left(\frac{1}{n} \sum_{i=1}^{n} h_{i i}^{r}\right) e_{r}
$$

and the squared norm of $h$ over dimension $n$ is denoted by $\mathcal{C}$ and is called the Casorati curvature of the submanifold $M$. Therefore we have

$$
\mathcal{C}=\frac{1}{n} \sum_{r=n+1}^{n+p} \sum_{i, j=1}^{n}\left(h_{i j}^{r}\right)^{2}
$$


Let $K\left(e_{i} \wedge e_{j}\right), 1 \leq i<j \leq n$, denote the sectional curvature of the plane section spanned by $e_{i}$ and $e_{j}$. Then the scalar curvature of $M^{n}$ is given by

$$
\tau=\sum_{i<j} K\left(e_{i} \wedge e_{j}\right)
$$

and the normalized scalar curvature $\rho$ is defined by

$$
\rho=\frac{2 \tau}{n(n-1)} .
$$

Suppose $L$ is an $l$-dimensional subspace of $T_{x} M, x \in M, l \geq 2$ and $\left\{e_{1}, \ldots, e_{l}\right\}$ an orthonormal basis of $L$. Then the scalar curvature $\tau(L)$ of the $l$-plane $L$ is given by

$$
\tau(L)=\sum_{1 \leq \mu<\nu \leq l} K\left(e_{\mu} \wedge e_{\nu}\right),
$$

and the Casorati curvature $\mathcal{C}(L)$ of the subspace $L$ is defined as

$$
\mathcal{C}(L)=\frac{1}{r} \sum_{r=n+1}^{n+p} \sum_{i, j=1}^{n}\left(h_{i j}^{r}\right)^{2} .
$$

For more details of slant submanifolds in quaternionic space forms, we refer to $[2,4]$.

\section{Optimization method on Riemannian submanifolds}

Let $\left(N_{2}, \bar{g}\right)$ be a Riemannian manifold, $N_{1}$ be a Riemannian submanifold of it, $g$ be the metric induced on $N_{1}$ by $\bar{g}$ and $f: N_{1} \rightarrow \mathbb{R}$ be a differentiable function.

Following [5-7] we considered the constrained extremum problem

$$
\min _{x \in N_{1}} f(x)
$$

then we have the following.

Lemma 1 ([5]) If $x_{0} \in N_{1}$ is the solution of the problem (4), then

(i) $(\operatorname{grad} f)\left(x_{0}\right) \in T_{x_{0}}^{\perp} N_{1}$;

(ii) the bilinear form

$$
\begin{aligned}
& \mathcal{A}: T_{x_{0}} N_{1} \times T_{x_{0}} N_{1} \rightarrow \mathbb{R} \\
& \mathcal{A}(X, Y)=\operatorname{Hess}_{f}(X, Y)+\bar{g}\left(h(X, Y),(\operatorname{grad} f)\left(x_{0}\right)\right)
\end{aligned}
$$

is positive semidefinite, where $h$ is the second fundamental form of $N_{1}$ in $N_{2}$.

In [6], the above lemma was successfully applied to improve an inequality relating $\delta(2)$ obtained in [8]. Later, Chen extended the improved inequality to the general inequalities involving $\delta$-invariants $\delta\left(n_{1}, \ldots, n_{k}\right)$ [9]. More details of $\delta$-invariants can be found in [1015]. Besides, the first author gave another proof of the inequalities relating the normalized $\delta$-Casorati curvature $\hat{\delta}_{c}(n-1)$ for submanifolds in real space forms by using T Oprea's optimization method [16]. 


\section{Proof of Theorem 1}

From the Gauss equation we can easily obtain (see (12) in [2])

$$
2 \tau=\frac{c}{4}\left[n(n-1)+9 n \cos ^{2} \theta\right]+n^{2}\|H\|^{2}-n \mathcal{C} .
$$

We define now the following function, denoted by $\mathcal{Q}$, which is a quadratic polynomial in the components of the second fundamental form:

$$
\mathcal{Q}=\frac{1}{2} n(n-1) \mathcal{C}+\frac{1}{2}(n+1)(n-1) \mathcal{C}(L)-2 \tau+\frac{c}{4}\left[n(n-1)+9 n \cos ^{2} \theta\right] .
$$

Without loss of generality, by assuming that $L$ is spanned by $e_{1}, \ldots, e_{n-1}$, one gets

$$
\mathcal{Q}=\frac{n+1}{2} \sum_{\alpha=n+1}^{4 m}\left[\sum_{i, j=1}^{n}\left(h_{i j}^{\alpha}\right)^{2}\right]+\frac{n+1}{2} \sum_{\alpha=n+1}^{4 m}\left[\sum_{i, j=1}^{n-1}\left(h_{i j}^{\alpha}\right)^{2}\right]-\sum_{\alpha=n+1}^{4 m}\left(\sum_{i=1}^{n} h_{i i}^{\alpha}\right)^{2},
$$

here we used (5) and (6).

From (7) we have

$$
\begin{aligned}
\mathcal{Q}= & \sum_{\alpha=n+1}^{4 m} \sum_{i=1}^{n-1}\left[n\left(h_{i i}^{\alpha}\right)^{2}+(n+1)\left(h_{i n}^{\alpha}\right)^{2}\right] \\
& +\sum_{\alpha=n+1}^{4 m}\left[2(n+1) \sum_{1 \leq i<j \leq n-1}\left(h_{i j}^{\alpha}\right)^{2}-2 \sum_{1 \leq i<j \leq n} h_{i i}^{\alpha} h_{j j}^{\alpha}+\frac{n-1}{2}\left(h_{n n}^{\alpha}\right)^{2}\right] \\
\geq & \sum_{\alpha=n+1}^{4 m} \sum_{i=1}^{n-1} n\left(h_{i i}^{\alpha}\right)^{2}+\sum_{\alpha=n+1}^{4 m}\left[-2 \sum_{1 \leq i<j \leq n} h_{i i}^{\alpha} h_{j j}^{\alpha}+\frac{n-1}{2}\left(h_{n n}^{\alpha}\right)^{2}\right] .
\end{aligned}
$$

For $\alpha=n+1, \ldots, 4 m$, let us consider the quadratic form

$$
\begin{aligned}
& f_{\alpha}: \mathbb{R}^{n} \rightarrow \mathbb{R} \\
& f_{\alpha}\left(h_{11}^{\alpha}, \ldots, h_{n n}^{\alpha}\right)=\sum_{i=1}^{n-1} n\left(h_{i i}^{\alpha}\right)^{2}-2 \sum_{1 \leq i<j \leq n} h_{i i}^{\alpha} h_{j j}^{\alpha}+\frac{n-1}{2}\left(h_{n n}^{\alpha}\right)^{2}
\end{aligned}
$$

and the constrained extremum problem

$\min f_{\alpha}$

subject to $\digamma: h_{11}^{\alpha}+\cdots+h_{n n}^{\alpha}=k^{\alpha}$,

where $k^{\alpha}$ is a real constant.

The partial derivatives of the function $f_{\alpha}$ are

$$
\begin{aligned}
& \frac{\partial f_{\alpha}}{\partial h_{11}^{\alpha}}=2 n h_{11}^{\alpha}-2 \sum_{i=2}^{n} h_{i i}^{\alpha}, \\
& \frac{\partial f_{\alpha}}{\partial h_{22}^{\alpha}}=2 n h_{22}^{\alpha}-2 h_{11}^{\alpha}-2 \sum_{i=3}^{n} h_{i i}^{\alpha},
\end{aligned}
$$




$$
\begin{aligned}
& \frac{\partial f_{\alpha}}{\partial h_{n-1, n-1}^{\alpha}}=2 n h_{n-1, n-1}^{\alpha}-2 \sum_{i=1}^{n-2} h_{i i}^{\alpha}-2 h_{n n}^{\alpha}, \\
& \frac{\partial f_{\alpha}}{\partial h_{n n}^{\alpha}}=-2 \sum_{i=1}^{n-1} h_{i i}^{\alpha}+(n-1) h_{n n}^{\alpha} .
\end{aligned}
$$

For an optimal solution $\left(h_{11}^{\alpha}, h_{22}^{\alpha}, \ldots, h_{n n}^{\alpha}\right)$ of the problem in question, the vector $\operatorname{grad} f_{\alpha}$ is normal at $\digamma$, that is, it is collinear with the vector $(1,1, \ldots, 1)$. From (9), (10), (11), and (12), it follows that a critical point of the considered problem has the form

$$
\left(h_{11}^{\alpha}, h_{22}^{\alpha}, \ldots, h_{n-1, n-1}^{\alpha}, h_{n n}^{\alpha}\right)=\left(t^{\alpha}, t^{\alpha}, \ldots, t^{\alpha}, 2 t^{\alpha}\right)
$$

As $\sum_{i=1}^{n} h_{i i}^{\alpha}=k^{\alpha}$, by using (13) we have

$$
h_{11}^{\alpha}=h_{22}^{\alpha}=\cdots=h_{n-1, n-1}^{\alpha}=\frac{1}{n+1} k^{\alpha}, \quad h_{n n}^{\alpha}=\frac{2}{n+1} k^{\alpha} .
$$

We fix an arbitrary point $x \in \digamma$. The 2-form $\mathcal{A}: T_{x} \digamma \times T_{x} \digamma \rightarrow \mathbb{R}$ has the expression

$$
\mathcal{A}(X, Y)=\operatorname{Hess} f_{\alpha}(X, Y)+\left\langle h^{\prime}(X, Y),\left(\operatorname{grad} f_{\alpha}\right)(x)\right\rangle
$$

where $h^{\prime}$ is the second fundamental form of $\digamma$ in $\mathbb{R}^{n}$ and $\langle$,$\rangle is the standard inner-product$ on $\mathbb{R}^{n}$. In the standard frame of $\mathbb{R}^{n}$, the Hessian of $f_{\alpha}$ has the matrix

$$
\left(\begin{array}{cccccc}
2 n & -2 & -2 & \cdots & -2 & -2 \\
-2 & 2 n & -2 & \cdots & -2 & -2 \\
-2 & -2 & 2 n & \cdots & -2 & -2 \\
\vdots & \vdots & \vdots & \ddots & \vdots & \vdots \\
-2 & -2 & -2 & \ldots & 2 n & -2 \\
-2 & -2 & -2 & \ldots & -2 & n-1
\end{array}\right)
$$

As $\digamma$ is totally geodesic in $\mathbb{R}^{n}$, considering a vector $X$ tangent to $\digamma$ at the arbitrary point $x$ on $\digamma$, that is, verifying the relation $\sum_{i=1}^{n} X_{i}=0$, we have

$$
\begin{aligned}
\mathcal{A}(X, X) & =\left(X_{1}, X_{2}, X_{3}, \ldots, X_{n-1}, X_{n}\right)\left(\begin{array}{cccccc}
2 n & -2 & -2 & \cdots & -2 & -2 \\
-2 & 2 n & -2 & \cdots & -2 & -2 \\
-2 & -2 & 2 n & \cdots & -2 & -2 \\
\vdots & \vdots & \vdots & \ddots & \vdots & \vdots \\
-2 & -2 & -2 & \cdots & 2 n & -2 \\
-2 & -2 & -2 & \cdots & -2 & n-1
\end{array}\right)\left(\begin{array}{c}
X_{1} \\
X_{2} \\
X_{3} \\
\vdots \\
X_{n-1} \\
X_{n}
\end{array}\right) \\
& =2(n+1) \sum_{i=1}^{n-1} X_{i}^{2}+(n+1) X_{n}^{2}-2\left(X_{1}+X_{2}+\cdots+X_{n}\right)^{2} \\
& =2(n+1) \sum_{i=1}^{n-1} X_{i}^{2}+(n+1) X_{n}^{2} \\
& \geq 0 .
\end{aligned}
$$


Thus the point $\left(h_{11}^{\alpha}, h_{22}^{\alpha}, \ldots, h_{n n}^{\alpha}\right)$ given by (14) is a global minimum point, here we used Lemma 1. Inserting (14) in (8) we have

$$
\mathcal{Q} \geq 0
$$

From (2), (6), and (15) we can derive inequality (3). The equality case of (3) holds if and only if we have the equality in all the previous inequalities. Thus

$$
\begin{aligned}
& h_{i j}^{\alpha}=0, \quad i \neq j, \forall \alpha ; \\
& h_{n n}^{\alpha}=2 h_{11}^{\alpha}=2 h_{22}^{\alpha}=\cdots=2 h_{n-1, n-1}^{\alpha}, \quad \forall \alpha .
\end{aligned}
$$

\section{Competing interests}

The authors declare that they have no competing interests.

\section{Authors' contributions}

All authors contributed equally and significantly in writing this paper. All authors read and approved the final manuscript.

\section{Acknowledgements}

We would like to thank to Professor Weidong Song, who has always been generous with his time and advice.

Received: 8 August 2014 Accepted: 27 October 2014 Published: 10 Nov 2014

\section{References}

1. Decu, S, Haesen, S, Verstraelen, L: Optimal inequalities involving Casorati curvatures. Bull. Transilv. Univ. Brasov Ser. B 14(49), suppl., 85-93 (2007)

2. Slesar, V, Sahin, B, Vîlcu, GE: Inequalities for the Casorati curvatures of slant submanifolds in quaternionic space forms. J. Inequal. Appl. 2014, Article ID 123 (2014)

3. Lee, CW, Yoon, DW, Lee, JW: Optimal inequalities for the Casorati curvatures of submanifolds of real space forms endowed with semi-symmetric metric connections. J. Inequal. Appl. 2014, Article ID 327 (2014)

4. Lee, JW, Vîlcu, GE: Inequalities for generalized normalized $\delta$-Casorati curvatures of slant submanifolds in quaternionic space forms (2014). arXiv:1405.5192v1 [math.DG]

5. Oprea, T: Optimization methods on Riemannian submanifolds. An. Univ. Bucur., Mat. 54, 127-136 (2005)

6. Oprea, T: Chen's inequality in the Lagrangian case. Colloq. Math. 108, 163-169 (2007)

7. Oprea, T: Ricci curvature of Lagrangian submanifolds in complex space forms. Math. Inequal. Appl. 13(4), 851-858 (2010)

8. Chen, B-Y, Dillen, F, Verstraelen, L, Vrancken, L: Totally real submanifolds of $\mathbb{C} P^{n}$ satisfying a basic equality. Arch. Math. 63, 553-564 (1994)

9. Chen, B-Y, Dillen, F: Optimal general inequalities for Lagrangian submanifolds in complex space forms. J. Math. Anal. Appl. 379(1), 229-239 (2011)

10. Chen, B-Y: Some pinching and classification theorems for minimal submanifolds. Arch. Math. 60, 568-578 (1993)

11. Al-Solamy, FR, Chen, B-Y, Deshmukh, S: Two optimal inequalities for anti-holomorphic submanifolds and their applications. Taiwan. J. Math. 18, 199-217 (2014)

12. Vîlcu, GE: On Chen invariant and inequalities in quaternionic geometry. J. Inequal. Appl. 2013, Article ID 66 (2013)

13. Gülbahar, M, Kılıç, E, Keles, S: Chen-like inequalities on lightlike hypersurfaces of a Lorentzian manifold. J. Inequal. Appl. 2013, Article ID 266 (2013)

14. Zhang, P, Zhang, L, Song, W: Chen's inequalities for submanifolds of a Riemannian manifold of quasi-constant curvature with a semi-symmetric metric connection. Taiwan. J. Math. (2014). doi:10.11650/tjm.18.2014.4045

15. Zhang, P: Remarks on Chen's inequalities for submanifolds of a Riemannian manifold of nearly quasi-constant curvature. Vietnam J. Math. (2014). doi:10.1007/s10013-014-0096-9

16. Zhang, P: Inequalities for Casorati curvatures of submanifolds in real space forms (2014). arXiv:1408.4996 [math.DG]

10.1186/1029-242X-2014-452

Cite this article as: Zhang and Zhang: Remarks on inequalities for the Casorati curvatures of slant submanifolds in quaternionic space forms. Journal of Inequalities and Applications 2014, 2014:452 